\title{
RECUPERACIÓN, REHABILITACIÓN Y PREVENCIÓN DE TRASTORNOS MUSCULOESQUELÉTICOS EN UNA PLANTA AUTOMOTRIZ
}

\author{
RECOVERY, REHABILITATION AND PREVENTION OF MUSCULOSKELETAL \\ DISORDERS IN AN AUTOMOTIVE PLANT
}

\author{
Martin Arturo Cisneros* \\ Alejandro Pabo López-Gaffney ${ }^{* *}$ \\ Carlos Alberto López ${ }^{* * *}$ \\ Julio Omar Amnuel ${ }^{* * * *}$ \\ Martin Leopoldo Musulin**** \\ Juan Pablo Jose Giménez ${ }^{* * * * * *}$ \\ Alejandro Miguel Alasino ${ }^{* * * * * *}$
}

\begin{abstract}
Resumen: El objetivo es realizar el relevamiento de los casos que requieren rehabilitación para volver a trabajar y recuperar las condiciones físicas de los trabajadores. Se realizó fortalecimiento por profesiograma, rehabilitación de las altas médicas y rehabilitación preventiva. La población de producción corresponde a 5.018 empleados usuarios del gimnasio de la empresa. La consulta al servicio involucra un interrogatorio médico y evaluación semiológica, medición de fuerza de prensión, y de rango de movimiento de las articulaciones. Las dolencias se tratan fortaleciendo los grupos musculares involucrados que estabilizan las articulaciones. Como resultado del estudio los médicos evaluadores de puestos adecuaron tareas, confeccionaron planillas individuales para registrar diagnóstico, situación clínica al comienzo, término y seguimiento posterior a los seis meses. Entre julio 2020 y marzo 2021 se trataron 199 casos con 93\% de retorno a tareas normales. Se constata la disminución de días de baja por enfermedad; la mayoría de los casos que son tratados en el gabinete son sin baja. Con la actividad del gabinete de rehabilitación el trabajador deja de tener molestias o dolencias, y se percibe que se siente cuidado y que su confianza en el servicio médico se incrementa.
\end{abstract}

Palabras clave: Rehabilitación, fábrica, gimnasia.

\footnotetext{
"Instituto Médico Laboral Zarate S.A. Campana, Argentina. Correo electrónico: cisnema9@gmail.com. Orcid: https://orcid.org/oooo-0002-8800-9565. Autor de correspondencia.

**Toyota Argentina. Pilar, Argentina. Correo electrónico: alejandrolopezgaffney@yahoo.com.ar. Orcid: https://orcid.org/oooo-0002-6694-3664

${ }^{* * *}$ Instituto Médico Laboral Zarate S.A. Zarate, Argentina. Correo electrónico: calop58@yahoo.com.ar. Orcid: https://orcid.org/oooo-0003-3153-3363

**** Instituto Médico Laboral Zarate S.A. Zarate, Argentina. Correo electrónico: jamnuelo@gmail.com. Orcid: https://orcid.org/oooo-0001-5209-4254

******Instituto Médico Laboral Zarate S.A. Zarate, Argentina. Correo electrónico: musulinmartin@gmail.com. Orcid: https://orcid.org/oooo-ooo2-1164-259X

*******Instituto Médico Laboral Zarate S.A. Zarate, Argentina. Correo electrónico: juampigimenez@hotmail.com. Orcid: https://orcid.org/oooo-0002-1175-4102

${ }_{* * * * * * * *}$ Instituto Médico Laboral Zarate S.A. Zarate, Argentina. Correo electrónico: alealasino68@gmail.com. Orcid: https://orcid.org/oooo-0002-9891-0891
} 


\begin{abstract}
The objective is to carry out the survey of the cases that require rehabilitation to return to work and recover the physical conditions of the workers. Strengthening by profesiogram, rehabilitation of medical discharges and preventive rehabilitation was carried out. The production population corresponds to 5,018 employee users of the company's gym. The consultation of the service involves a medical questioning and semiological evaluation, measurement of grip strength, and range of motion of the joints. Ailments are treated by strengthening the muscle groups involved that stabilize the joints. As a result of the study, the evaluating doctors adapted tasks, prepared individual forms to record diagnosis, clinical situation at the beginning, end, and follow-up after six months. Between july 2020 and march 2021, 199 cases were treated with 93\% return to normal tasks. There is a decrease in days of sick leave; most of the cases that are treated in the cabinet are without sick leave. The rehabilitation office's activity makes the worker feel cared for, the discomfort or ailments disappear and their confidence in the medical service increases.
\end{abstract}

Keywords: Rehabilitation, factory, gymnastics.

Recepción: 09.06.2021 / Revisión: 02.09.2021 / Aceptación: 19.10.2021

\title{
Introducción
}

Se realiza un estudio en una planta automotriz, sucursal de una empresa multinacional, ubicada en Zarate, provincia de Buenos Aires de la República Argentina. Esta planta comienza a producir en el año 1997, cuenta con una superficie cubierta de 282.000 metros cuadrados, y alrededor de 6060 empleados, de los cuales unos 5078, están dedicados a la producción. La sucursal cuenta con empresas contratistas que desarrollan sus actividades en la planta y algunas fuera de ella, prestando servicios secundarios como limpieza, comedores y servicio médico.

La producción se lleva a cabo en dos turnos y con una capacidad máxima para producir un vehículo cada 90 segundos, lo que equivale a 614 unidades por día. Para lograr este objetivo sostiene una organización rigurosa del trabajo. En esta organización de trabajo, la actividad laboral es secuenciada, prescripta e inequívoca. Llevada adelante en el espacio destinado a ella y siguiendo el ritmo de la velocidad de la línea de producción. Este sistema organiza células de tareas de 4 a 6 personas que se reparten operaciones para ir armando un vehículo. Cada puesto debe ser ocupado por un colaborador que cumpla con los criterios antropométricos preestablecidos por análisis ergonómicos. Se define un profesiograma para cada sector por plano de trabajo y tipo de tarea; es decir, un rango de estatura y un mínimo de fuerza de prensión de ambas manos.

La finalización de la labor de una célula encastra en forma inmediata con la siguiente. Las habilidades y conocimientos de los trabajadores de una célula son parecidos a la siguiente; pero no son válidas en otro sector. Al ser un sistema de producción Just in Time, no existe la posibilidad de almacenar piezas terminadas y se trabaja con el mínimo indispensable de personal. Se sigue el concepto de fábrica flaca, sin acumular insumos (Coriat, 1992). Cada una de estas células cuenta con un Team Leader que cubre el puesto cuando un operario discontinúa su labor, y también se dispone de los denominados "cubre ausentes", que son operarios de experiencia que pueden hacer todas las operaciones. 
En la línea de producción el trabajador no es dueño de la posibilidad de descansar o bajar el ritmo de actividad. El cansancio es la falta de fuerza que resulta de haberse fatigado. También es el hastío, tedio o fastidio que genera el trabajo. Esta sensación está ligada a los turnos rotativos, por los que no se logra descansar apropiadamente. La fatiga debe considerarse como un estado moderador de la actividad, es la incapacidad para seguir generando un nivel de fuerza o una intensidad de ejercicio determinada. Se trata de un problema tanto central como periférico. Esta incapacidad de mantener la generación de fuerzas a nivel central es producto de la información que recibe la corteza cerebral de las aferencias periféricas. La aparición de la fatiga logra disminuir las acciones ejecutadas en cualquier actividad. La edad aumenta la susceptibilidad a la fatiga (Moreno-Quinchanegua, 2017; Arce-Rodríguez, 2015).

Los trabajadores expresan en la consulta la aparición de molestias musculoesqueléticas. Generalmente es una descripción inespecífica en el segmento comprometido, solicitan un analgésico; la evaluación semiológica no da signos y síntomas de una enfermedad. El trabajador no percibe aún la incapacidad funcional en el proceso de gestación.

En la siguiente consulta si no se intervino antes, el trabajador relatará la aparición de dolor, la evaluación semiológica ya tendrá mayor consistencia, la acción involucrada, el grupo muscular y las articulaciones comprometidas. Muchas veces se interpreta como una sobrecarga, cuando están involucrados los músculos que realizan la acción. Otra posibilidad es la aparición de contractura muscular, más habitual de los músculos que mantienen la postura. Aún no está limitada la realización de los movimientos. Esto se asociará a la inmediata limitación de la función y se tendrá el cuadro clínico de una enfermedad.

Figura 1. Secuencia observada desde el servicio médico.

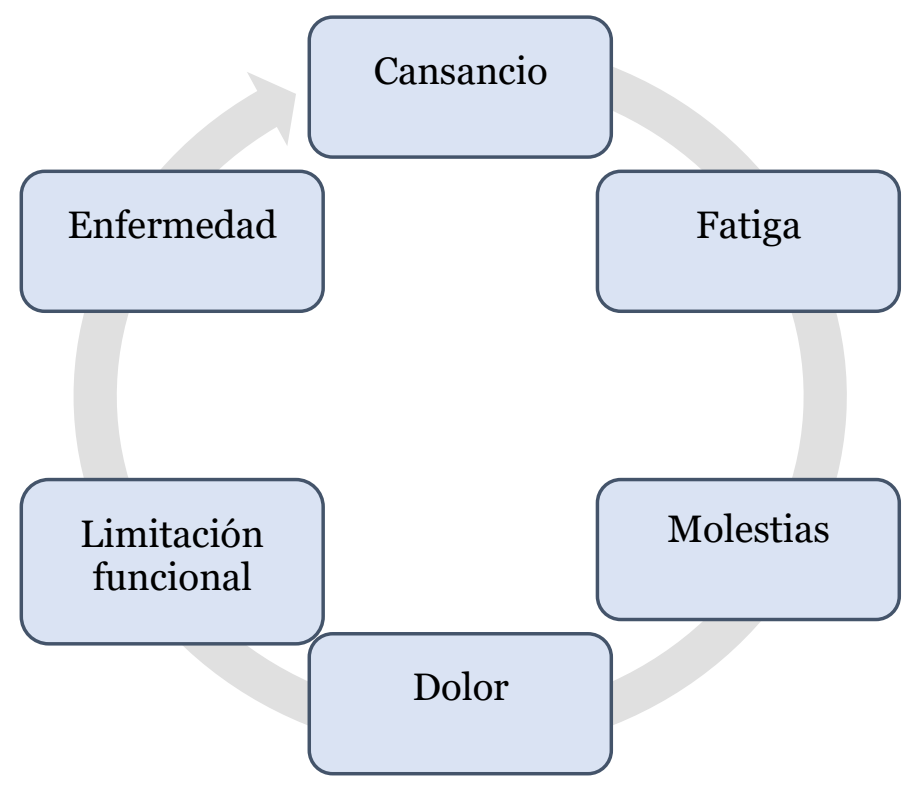

Si la mencionada enfermedad forma parte del listado de enfermedades profesionales se trata entonces de una enfermedad profesional y será derivado a la Aseguradora de Riesgo de Trabajo (ART), caso contrario será derivado por la obra social. 
Con el alta el trabajador habrá dejado de tener dolor, pero la inactividad lo ha debilitado. Si requirió inmovilización, las funciones del segmento tienen mayor deterioro. Por otro lado, la memoria del dolor lo llevará a limitar involuntariamente la actividad del segmento enfermo, compensando esta inacción con el segmento contralateral y los músculos antagonistas.

Los miembros de la célula son monitoreados en sus actividades, tanto en sus tiempos de producción y calidad de esta, como también en sus movimientos, sus posturas y las acciones desarrolladas durante el trabajo. Este monitoreo es llevado adelante por los supervisores, por personal de higiene y seguridad industrial, por ergónomos y médicos evaluadores de puestos. Esta acción de observar al trabajador para mejorar posturas y gestos en su lugar de trabajo comenzó en Inglaterra en la década de 1920. Durante 1923 en el Reino Unido comenzó a trabajar el fisioterapeuta en la industria, pero no fue hasta la segunda guerra mundial, con sus consecuentes cambios en la industria, tecnología, ciencia y la aparición del ímpetu por el desarrollo ergonómico, que alcanzan protagonismo. Inicialmente, sus funciones estaban relacionadas con el tratamiento y la rehabilitación de los trabajadores, y fueron preparándose para ser capaces de desarrollar prevención de riesgos laborales. En Finlandia los fisioterapeutas se incorporan a la salud ocupacional en 1950 y en Suecia en 1965 (Mugueta - Aguinaga et al., 2015).

La industria automotriz de diferentes países europeos incorporó esta actividad preventiva y de rehabilitación por medio de los fisioterapeutas y ergónomos. En Suecia, Volvo y Ericsson innovaron en la actividad incorporando la "pausa para la gimnasia", que evolucionó a ejercicios para relajar la musculatura. Actualmente se denomina "pausas activas”. En Latinoamérica, Brasil con la resolución 259 de diciembre de 2003, determinó que el fisioterapeuta tiene poder para dar asistencia en la prevención de la salud de los trabajadores y puede contribuir legalmente con acciones que promocionen, prevengan y recuperen esta salud. En el país mencionado existe la especialidad fisioterapeuta del trabajo (Mugueta-Aguinaga et al., 2015).

\section{Pandemia 2020, sus consecuencias en el ámbito laboral}

El 11 de marzo de 2020 la enfermedad causada por el SARS-CoV-2 fue reconocida como pandemia por la Organización Mundial de la Salud. En Argentina, el primer caso se detecta el 3 de marzo de 2020. El 20 marzo de 2020 entra en vigencia la cuarentena obligatoria para la población argentina, con excepción de trabajadores considerados esenciales (Poder Ejecutivo Nacional, 2020, 20 de marzo). La pandemia genera el cierre de actividades por tres meses con el consecuente aislamiento obligatorio domiciliario del personal. Esta situación lleva a gran parte de los trabajadores de la planta automotriz a la pérdida de entrenamiento, y resta habilidad manual; asociándose a alteraciones del sueño y hábitos alimentarios, y con la imposibilidad de desarrollar actividades recreativas o deportivas, se degradan sus relaciones, poniendo en riesgo su integridad psicosocial.

Como refleja el informe del Observatorio Psicología Social Aplicada de la Facultad de Psicología de la Universidad de Bueno Aires (UBA), que realiza un muestreo incidental y estratificado en el país a los 7-11 días, 50-55 días y 115-124 días de cuarentena obligatoria, en el que se demuestra un incremento de porcentaje de la población con riesgo de trastorno 
mental, encuentra que menos de la mitad de los participantes realiza actividades protectoras como actividad física, meditación, práctica religiosa o yoga. Se incrementa en forma sostenida el uso de medicación automedicada, se demuestra que un porcentaje superior al 70\% de participantes sufren de alteraciones del sueño. Así como también el incremento del tabaquismo y del consumo de alcohol de $37,5 \%$ en el primer periodo, a 54,2\% al último periodo estudiado, y del uso de drogas ilegales de 10,45\% a 12,13 \% (Etchevers et al., 2020).

Los servicios de salud se enfocaron en los casos de COVID-19, manteniéndose cerrados los centros de fisio kinesioterapia o de rehabilitación, así como los gimnasios. En el momento de cerrar las actividades no esenciales, trabajadores de la planta automotriz estaban operados de lesiones osteomusculares como meniscectomías, lesiones de ligamento cruzado, de codo y hombros, tanto por obras sociales como por ART, quienes no podían realizar la rehabilitación requerida. Por las disposiciones del poder ejecutivo nacional las personas solo podían trasladarse al trabajo y a negocios de cercanía al pasar a distinta fase. Dado el cambio de fase de cuarentena, se logra dar comienzo a la producción en un turno y meses posteriores al segundo turno.

Para dar solución a estos problemas, se genera el proyecto de crear un gabinete de rehabilitación dentro del servicio médico, dirigido por la gerencia médica, formado por médicos evaluadores de puesto, el traumatólogo y profesores de educación física. Así también, se convoca a los kinesiólogos del staff, para que den soporte en los procesos terapéuticos del gabinete. El proyecto es presentado y aprobado por la dirección de la compañía y la entidad gremial. Se abre el gimnasio de la empresa como centro de rehabilitación, antes usado como lugar de entrenamiento personal, y en algunos casos, para tratar dolencias.

\section{Objetivo general}

Realizar con el equipo multidisciplinario del Departamento de Salud Ocupacional, el relevamiento de los casos que requieren rehabilitación para volver a trabajar y verificar las condiciones físicas de los trabajadores que estaban en tareas después de tres meses de inactividad, y por medio de terapia y acondicionamiento físico en el lugar de trabajo, generar su vuelta a tareas normales o habituales, en una planta automotriz de la ciudad de Zarate, Provincia de Buenos Aires.

\section{Objetivos específicos}

- Uniformar y registrar metódicamente por parte de los colaboradores del gabinete los rangos de movimiento y fuerza de los pacientes.

- Generar una forma de comunicación clara entre los actores sobre los casos tratados, dando lugar al registro de las observaciones de los miembros del gabinete.

- Disminución de días de baja por enfermedad y su consecuencia económica al evitar contratar reemplazos.

- Prevenir enfermedades profesionales.

- Mejorar la relación médico paciente. 


\section{Materiales y métodos}

\section{Diseño}

La selección de casos para ingresar al gabinete abarcó tres áreas:

\section{Fortalecimiento por profesiograma}

Llevar a los operarios asintomáticos a la fuerza requerida. Luego de un periodo de inactividad de dos meses, se hicieron mediciones de fuerza de prensión, hallando que un $20 \%$ de los operarios estaban por debajo de la fuerza que demanda su sector. Si bien, el trabajo propiamente dicho recupera fuerza necesaria en un $15 \%$ de este grupo, existe un porcentaje mayor que necesita ayuda. En las primeras dos semanas de fortalecimiento en gimnasio se dio de alta, considerándolo recuperado, al 65\%. El 20\% restante requirió dos semanas más para la recuperación.

\section{Rehabilitación de las altas médicas}

Los trabajadores de alta osteomusculares inculpables o de ART, fueron sometidos a un fortalecimiento posterior y rehabilitación de movilidad con el objetivo de no volver a enfermar. Al momento del alta el médico interviniente considera que la patología se ha curado o que la cirugía reparó lo dañado, y le da alta laboral. Pero desde la medicina laboral se debe evaluar si el trabajador realmente puede realizar sus tareas. Las ART no determinan el nivel de fuerza antes del alta y muchas veces tampoco el rango de movilidad.

\section{Rehabilitación preventiva}

Se intenta detener la secuencia: cansancio, fatiga, molestias, dolor, limitación de la función, enfermedad. Se trata de un círculo vicioso. Orientó a la rehabilitación identificar el estadio clínico y relacionar la clínica con la actividad laboral.

\section{Población}

La población del personal de producción de la fábrica al momento del estudio era de 5.018 personas, con un promedio de edad de 30 años. La mayoría ingresó entre los 18 y 20 años, y son del género masculino con estudios secundarios completos.

\section{Entorno}

El gimnasio de la empresa tiene 169 metros cuadrados, está equipado con 25 máquinas, y cuenta con un máximo de 8 estaciones de trabajo habilitadas por hora, de las cuales solo tres podían ser para patologías de rodilla. Se adquirieron materiales para rehabilitación de mano. Se realizaron dos limpiezas completas del gimnasio por turno, y se mantuvo circulación de aire con puertas y ventanas abiertas. Las actividades fueron realizadas con barbijos.

Los trabajadores en su horario de trabajo concurrían al gimnasio, se cambiaban de ropa, y luego procedían a un calentamiento lento según lo indicado por los médicos. Los profesores de educación física generaron un plan de entrenamiento llevando al equilibrio muscular involucrado en la estabilidad de las articulaciones. Específicamente los profesores de educación física buscaron brindar una formación física básica (FFB), con el objetivo de mejorar la performance física de los operarios cuando desarrollaban su actividad, atendiendo 
las deficiencias y patologías detectadas por el servicio médico. Con esta FFB los empleados pueden prevenir lesiones o recuperarse de las mismas mediante un trabajo de resistencia de fuerza si se habla de prevención, y protocolos adaptados (rutinas de ejercicio), si es que se habla de recuperación. Además, la FFB aporta la capacidad de contar con las cualidades de fuerza, movilidad articular, flexibilidad y coordinación para desempeñarse en tareas laborales o de la vida cotidiana de una manera eficaz.

La posibilidad de compartir los horarios de trabajo obligó a enfocarse en una correcta dosificación de las cargas para que el empleado pueda retornar a las actividades laborales y convivir con el plan de entrenamiento en el gimnasio. Otro aspecto importante para destacar son las actividades de recuperación realizadas dentro de la jornada laboral, como pausas activas en el lugar de trabajo.

\section{Intervenciones}

En la consulta al servicio médico por dolencias osteomusculares, se procede a un interrogatorio médico y evaluación semiológica. Formada una primera impresión se procede a:

- Medición de fuerza de prensión con dinamómetros de mano.

- Evaluación de rango de movimiento usando goniómetros adecuados a las articulaciones.

- Medición del diámetro de las extremidades en forma comparativa y luego en forma de seguimiento de la evolución de las actividades de fortalecimiento.

- En gimnasio, elongación de grupos musculares agonistas y antagonistas. Rehabilitación de rango movimiento.

- Fortalecimiento de los grupos musculares involucrados y de los que le dan estabilidad a las articulaciones.

- De requerirse, en casos de inmovilidad prolongada, uso de electroestimulación seguido de fortalecimiento y elongación. Proceder que disminuye los tiempos de recuperación de fuerza, que requiere coordinación entre kinesiólogo y el profesor de educación física.

- Orientación higiénico-dietética para generación de tejido muscular.

- En general solo se usó terapia física, no se involucraba la administración de analgésicos.

- En la planta, los médicos evaluadores de puestos restringieron algunas actividades laborales por la exposición que involucra esa tarea. Se adaptaron tareas, y se sumaron de ser posible, otras tareas u operaciones de menor exposición del segmento o articulación en rehabilitación.

- Con el trabajador presente en fábrica, realizando sus tareas o una adecuación temporal de tareas, se rehabilitó durante su jornada, retirándose de producción una hora y poniéndolo bajo la instrucción del profesor de educación física. Las tareas de 
elongación fueron realizadas a diario, y los fortalecimientos día por medio para no generar mayor fatiga. El día que no se entrenó el trabajador descansó sentado en el mitin o elongó en el gimnasio.

\section{Análisis estadístico}

La derivación a la rehabilitación se realizó con una orden médica y se completó una planilla individual confeccionada usando los gráficos del libro de goniometría de Taboadela (2007). Los integrantes del gabinete se comunicaron por esta vía, ya que es una documentación compartida en la intranet empresarial por los miembros.

A modo de ejemplo, en la figura A1 del anexo se observa la ficha de rehabilitación para patología o dolencia de hombro. En esta se registran los datos individuales, tales como el nombre, legajo, sector, tipo de patología (inculpable o laboral), lateralidad, dominancia, fuerza de prensión, impresión al ingreso y las observaciones, donde se escribió lo relacionado a esta dolencia, desde los estudios complementarios aportados, así como la evolución del estado clínico. Con las gráficas se recuerda cómo se realiza la goniometría y los valores de incapacidad nula.

$\mathrm{Al}$ mismo tiempo en una planilla de presentismo, se reflejaron sintéticamente los datos de la planilla individual, generando así una base de datos y por medio de una tabla dinámica, se generó la estadística de este artículo.

\section{Resultados y discusión}

Se trataron 199 casos desde julio 2020 hasta segunda semana de marzo de 2021.

Figura 2. Origen de las derivaciones al gabinete.

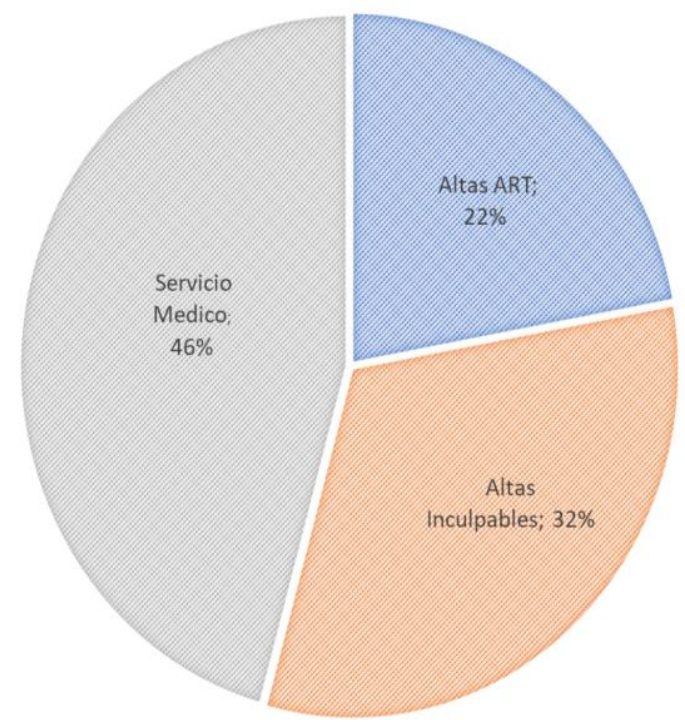

En la figura 3 se describen las patologías tratadas por regiones corporales; se destacan la contractura muscular (CM) de columna y en miembros superiores tendinopatía del supraespinoso (hombro) y la fatiga muscular. 
Figura 3. Patologías tratadas por regiones corporales.

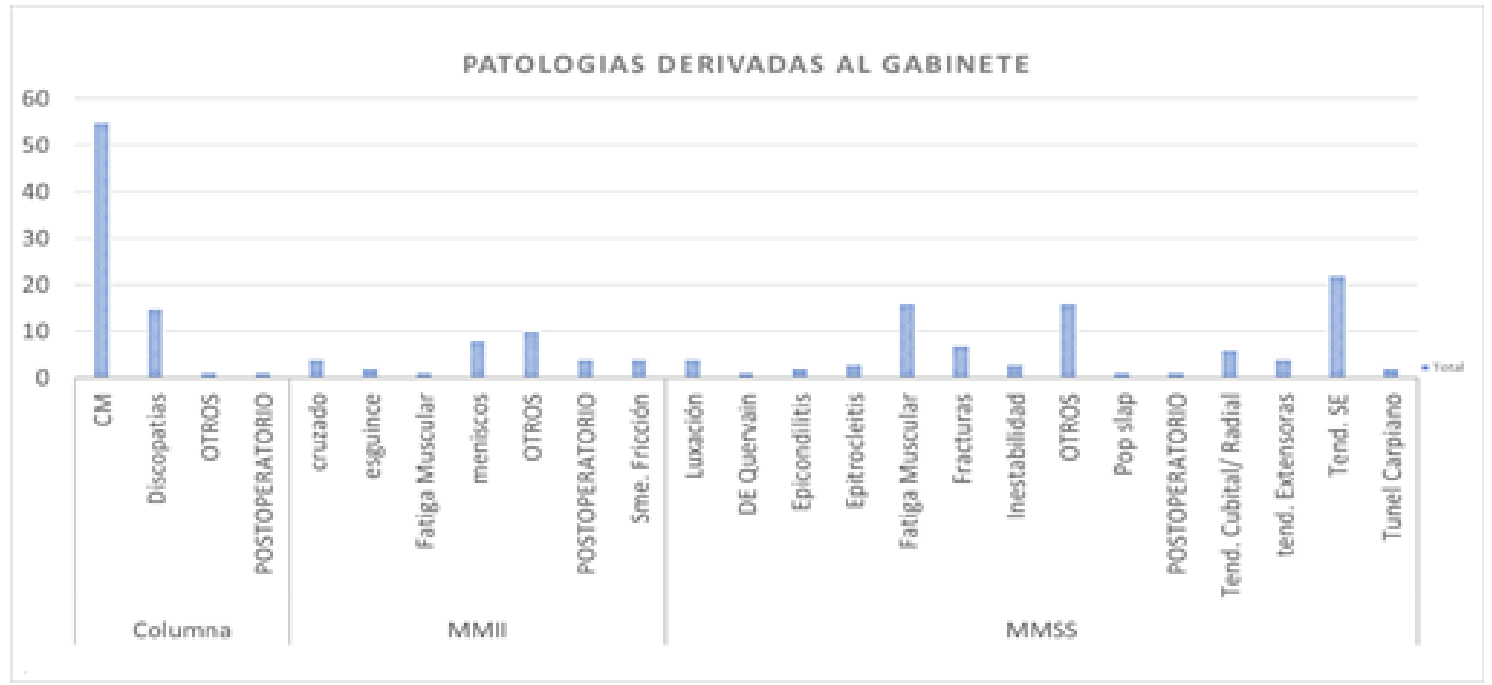

En las siguientes tres figuras se diferencian las regiones tratadas según origen.

Figura 4. Derivaciones desde servicio médico.

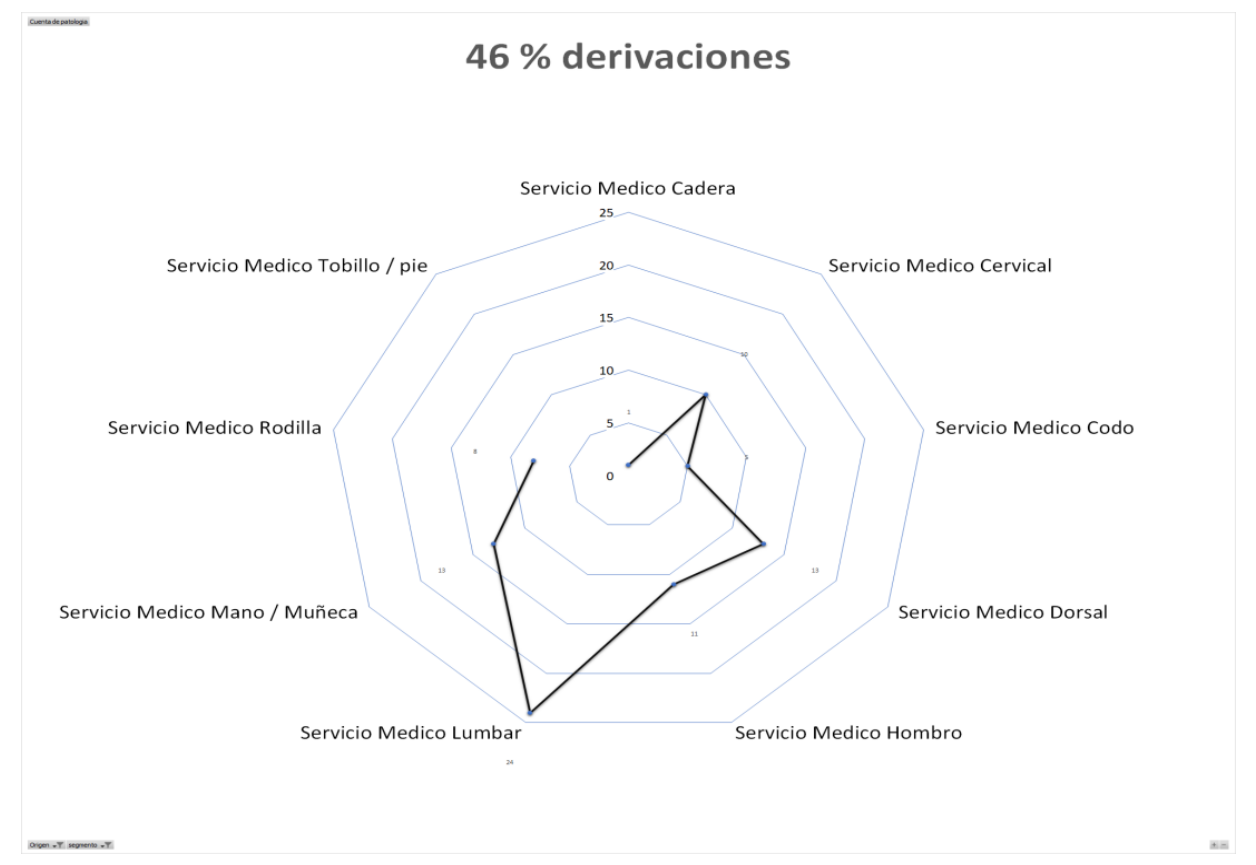


Figura 5. Derivaciones por altas médicas de la ART.

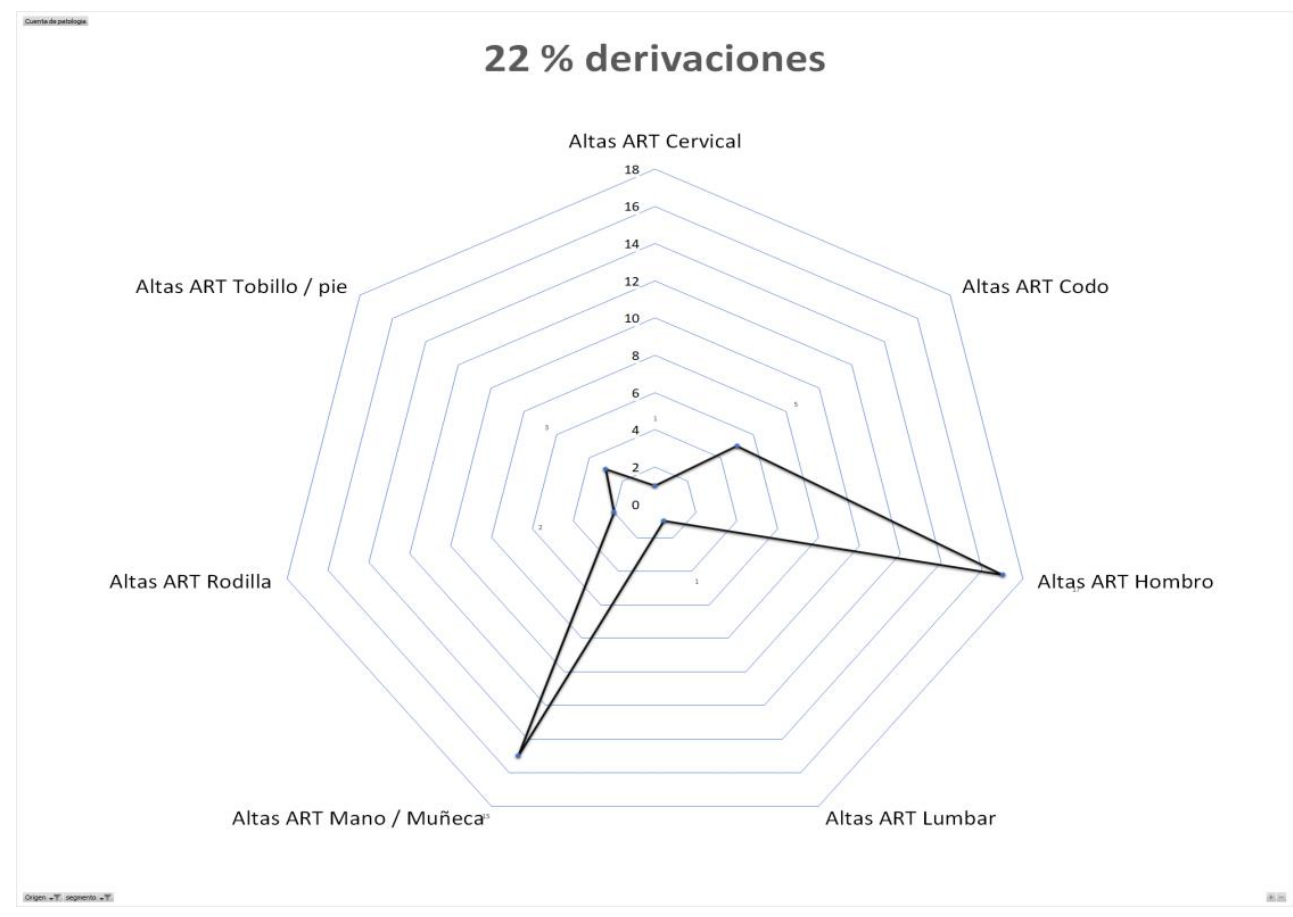

Figura 6. Derivaciones por altas de patología inculpables.

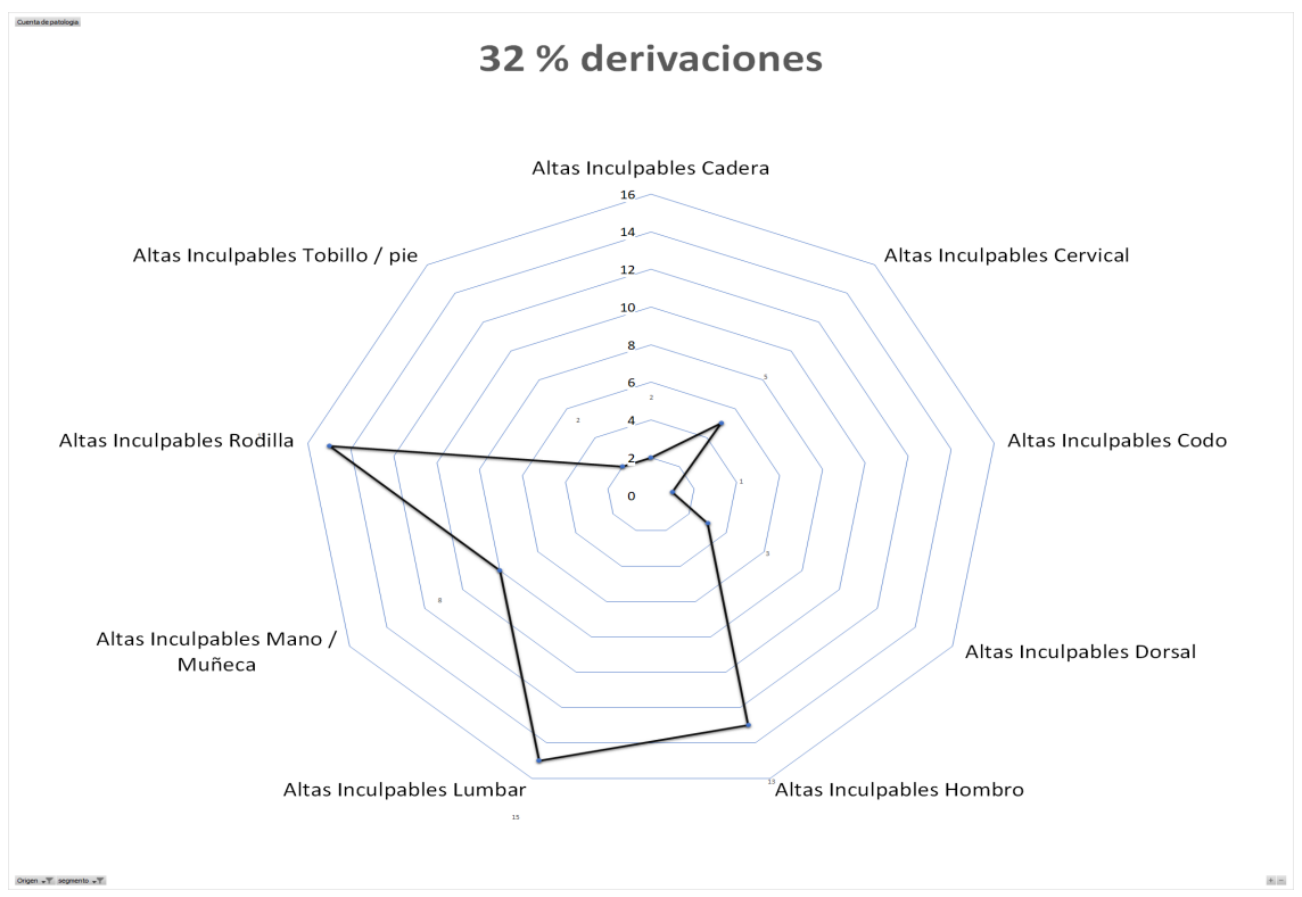


La estadística generada por las derivaciones a la rehabilitación de los trabajadores de los diferentes sectores demuestra a la vez las diferentes exposiciones a movimiento repetitivo o posiciones forzadas de la actividad laboral. Por ejemplo, la rehabilitación de columna y hombros se requiere en el sector Trim donde el trabajador ingresa a la estructura de la camioneta y trabaja en el armado del techo de la camioneta.

La figura 7 muestra la prevalencia de los segmentos corporales que generan síntomas y consultas por cada actividad. Los sectores de ensamble Final y Trim tienen exigencias de columna y de miembros superiores (MMSS). En cuanto al Material Handling (MH) o movimiento de materiales, se manejan carros eléctricos que descienden, y realizan carga y descarga de materiales, con mayor exigencia en miembros inferiores. En motores, existen netamente requerimientos de MMSS, ya que se trabaja erguido y no se requiere flexo extensión de columna lumbar. En soldadura hay demandas de columna cervical y lumbar asociadas a de MMSS.

Figura 7. Prevalencia de los segmentos corporales que generan síntomas y consultas por cada actividad.

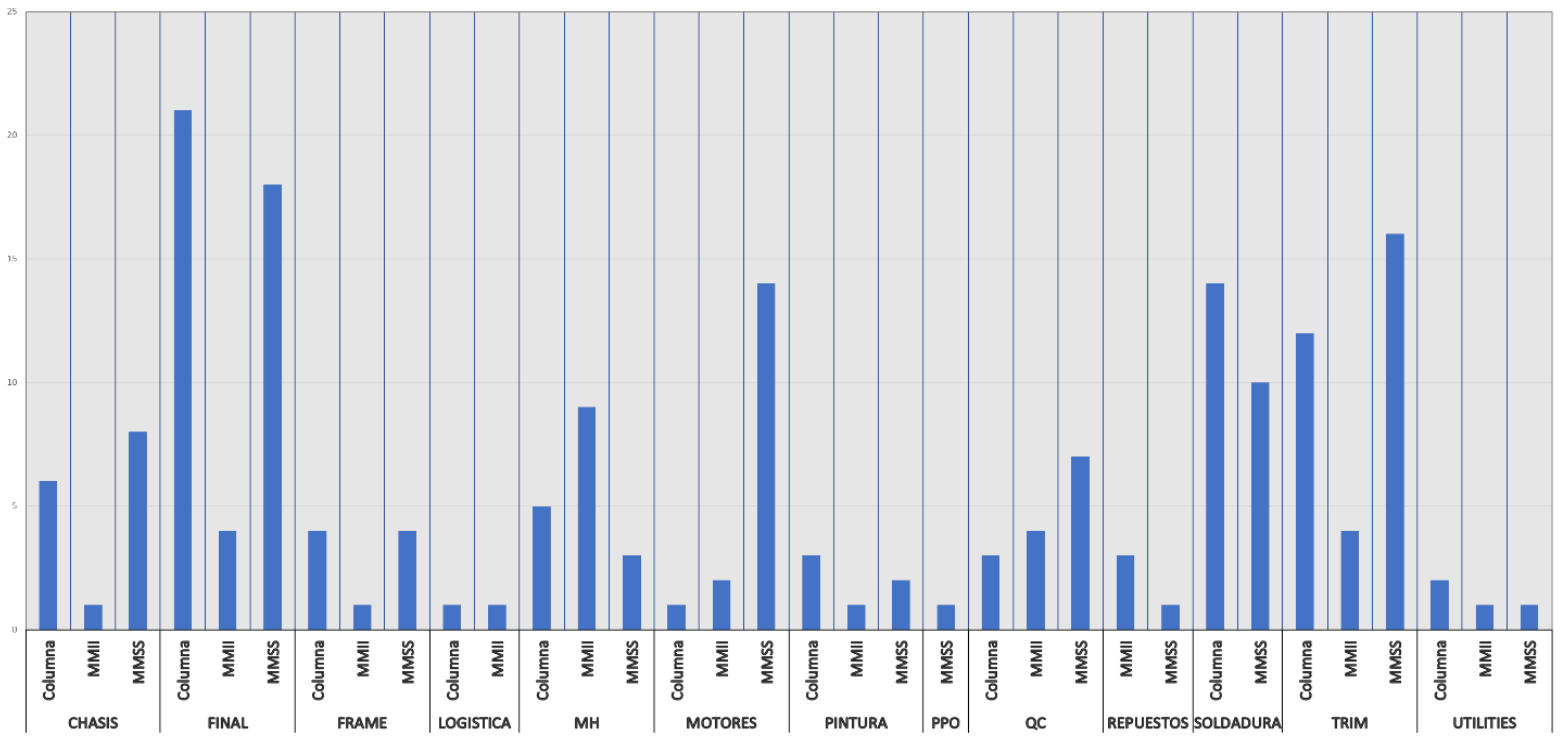

La figura 8 muestra las distintas patologías de MMSS derivadas al gabinete. La fatiga muscular y tendinopatías del supraespinoso (SE) son los motivos más frecuentes. 
Figura 8. Número de patologías de miembros superiores derivadas al gabinete.

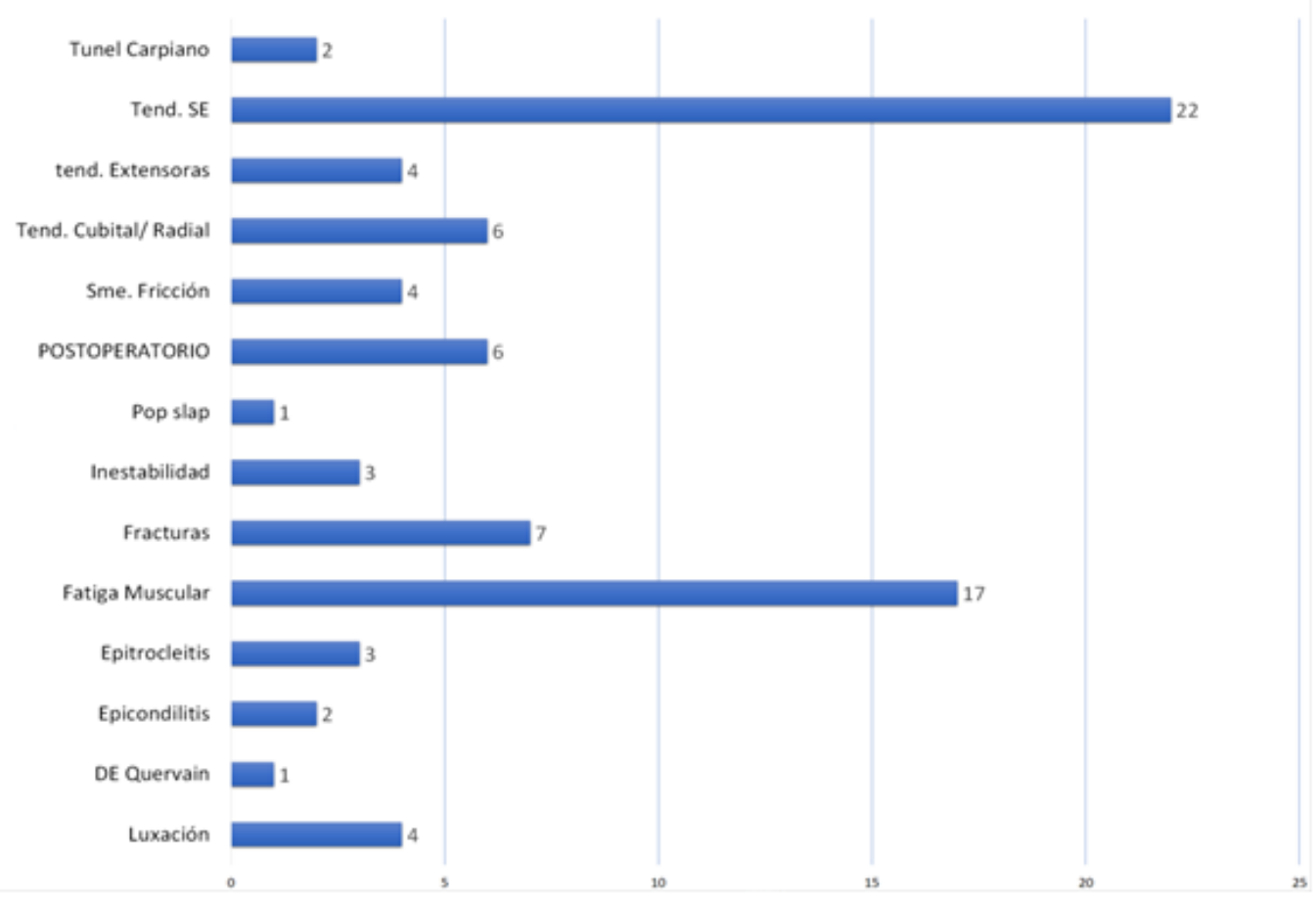

Las tareas de fortalecimiento según profesiograma se cumplieron en dos y cuatro semanas. En seguimiento a los seis meses, no se presentaron enfermedades musculoesqueléticas de miembros superiores.

En general, las rehabilitaciones de los hombros llevan un promedio de 9 sesiones. La tendinopatía sintomática del supraespinoso lleva un promedio 7,2 clases de rehabilitación. Se tuvo un caso de fractura de clavícula con inmovilización de un mes que requirió 27 clases para recuperar rango fisiológico de movimiento, llegando a realizar tareas habituales y vida normal fuera del trabajo. Las patologías de hombro llevaron a reubicar en el sector a 6 casos, realizando sus tareas normales.

Las lumbalgias sumaron 40 derivaciones, 322 clases con un promedio de 8,2. En tres casos se adecuaron tareas temporalmente, tres casos terminaron siendo reubicados, y hubo cuatro bajas por enfermedad. La edad promedio de los trabajadores fue de 31,5 años.

En general, fueron tratados 199 casos desde julio 2020 a marzo 2021, de los cuales:

- 173 desempeñaron tareas normales o habituales. Solo 15 casos adecuaron tareas temporalmente, con tareas normales al terminar el proceso.

- 13 casos requirieron reubicaciones, realizando todas las tareas en los nuevos puestos.

- Hubo 2 reingresos a ART de 44 altas ART, 2 derivaciones ART, y 9 casos de baja por enfermedad.

\section{Discusión}

La articulación del hombro es la más compleja del sistema osteoarticular y se fija al esqueleto por medio de la articulación esternoclavicular. Por ser un sistema articular complejo, es 
importante el fortalecimiento de los músculos en conjunto, equilibrando las tensiones entre agonistas y antagonistas, a fin de mantener la posición relativa de esta articulación, y corregir o asegurar el buen posicionamiento de la cabeza humeral en los centros de rotación instantáneos, evitando así la generación de síntomas como omalgias, por el uso que tiene en el trabajo. Por otro lado, se busca el fortalecimiento de los músculos que descienden la cabeza humeral, a fin de darle mayor espacio al supraespinoso y disminuir el conflicto de los movimientos de hombro en relación al ligamento coraco-acromial (Dufour \& Pillu, 2018).

Las lumbalgias son la principal derivación a gabinete de rehabilitación sin patología demostrada y la segunda causa de ausentismo después de las omalgias (Pinchao \& AguadoBalanta, 2019). Como factores que colaboran en la generación de lumbalgias, se menciona en primer lugar, que al tratarse de una población joven que promedia los 30 años, tienen como divertimento social y práctica deportiva el fútbol y correr; dos actividades que sobrecargan de impactos y esfuerzos a la columna.

La mayoría no entrena deporte, fútbol o atletismo; lo juega, y no se menciona en los interrogatorios una educación deportiva. Por lo cual, deberían entrar en calor, involucrando la movilidad de las articulaciones, la elongación de los grupos musculares a exigir, calentamiento de estos, y posterior a la actividad (por ejemplo, partido de fútbol), una bajada post actividad. No realizan ejercicios regenerativos después de las actividades. Esta información surge de los interrogatorios médicos durante las consultas de los trabajadores.

Esta falta de educación física genera isquiotibiales acortados funcionalmente. Este acortamiento tracciona desde sus inserciones isquiáticas provocando una anteversión de la pelvis con un pinzamiento posterior lumbosacro, rectificando la columna, y con ello, la exigencia de las carillas articulares interapofisarias, asociado a contractura de los glúteos, completando así la cadena posterior (Dufour \& Pillu, 2018).

Segundo factor es lo laboral, ya que los puestos de trabajo involucran bipedestación prolongada, aunque no estática.

Y como tercer factor está el sobrepeso y debilidad de los músculos anteriores del abdomen, que suman esfuerzo a los músculos posturales de la columna por ausencia funcional de la cincha abdominal.

Los cuadros de lumbalgia constituyen una fuente de ausentismo considerable, con examen físico sin signos patognomónicos de lesiones de columna o radiculopatías.

En el periodo agudo, de ser necesario, se indica reposo laboral, deportivo y antiinflamatorios no esteroideos; en cuanto cede el cuadro de dolor lumbar, se orienta a la rehabilitación temprana; en horario de trabajo y de ser necesario con adaptación de las tareas, restringiendo actividades de exigencia lumbar.

La rehabilitación consiste en fisio kinesioterapia y en gimnasio elongación muscular, tanto isquiotibial como lumbar. Luego, fortalecimiento de los músculos abdominales y lumbares. En algunos casos se requieren ejercicios de reeducación postural. También se sugiere la consulta al nutricionista para combatir el sobrepeso.

No se coincide con el trabajo de González-Gutiérrez et al. (2019), ya que se relaciona 
desde la clínica previo a la rehabilitación por lumbalgia el acortamiento funcional de los músculos isquiotibiales y en la evaluación de alta. Surge entonces investigar a futuro esta relación del acortamiento isquiotibial y la lumbalgia baja por medio del test distancia dedo piso en la población estudiada.

\section{Conclusiones}

Se concluye que el regreso a las tareas normales o habituales se cumple en un porcentaje superior al $93 \%$ de los casos. Se creó la ficha individual y se agregaron datos propios a la planilla de presentismo, gracias a lo que es posible un seguimiento individual y grupal, por región anatómica, por enfermedad, sector, edad, etc. Al mismo tiempo, se agrega la ficha la comunicación entre los miembros del gabinete.

Se constata la disminución de días de baja por enfermedad; la mayoría de los casos que son tratados en el gabinete son sin baja. De este modo se evitan las enfermedades profesionales, que de no intervenir se producirían.

Con la actividad del gabinete de rehabilitación, el trabajador deja de tener molestias o dolencias, y se percibe que se siente cuidado y que su confianza en el servicio médico se incrementa. Cabe mencionar la mejora en la comunicación con los diversos sectores de producción, al compartir la información estadística de los casos tratados.

Se concreta la oportunidad para dar respuestas simples en forma multidisciplinaria y holística a una compleja problemática. La etiología de las enfermedades es multifactorial, entonces la evaluación médica es caso a caso, la evaluación del puesto y la restricción temporaria de alguna actividad disminuye el riesgo de patología. Por otra parte, se realiza una actividad concreta en favor del presentismo. 


\section{Referencias}

Arce-Rodríguez, E. (2015). Mecanismos fisiológicos de la fatiga neuromuscular. Revista Médica de Costa rica y Centroamérica LXXII, (615), 461-464. https://www.medigraphic.com/pdfs/revmedcoscen/rmc-2015/rmc152zq.pdf

Coriat, B. (1992). Pensar al revés. Trabajo y organización en la empresa japonesa. Siglo XXI editores.

Dufour, M., \& Pillu, M. (2018). Biomecánica funcional. Miembros, cabeza y tronco. Elsevier.

Etchevers, M. J., Garay, C. J., Putrino, N., Grasso, J., Natalí, V., \& Helmich, N. (2020). Salud mental en cuarentena. Relevamiento del impacto psicológico a los 7-11, $50-55$ y 115-124 días de cuarentena en población argentina. Observatorio de Psicología Social Aplicada, Facultad de Psicología, Universidad de Buenos Aires. http://www.psi.uba.ar/opsa/\#informes González-Gutiérrez, M., León-Alava, O., Fuentes-Olea, A., Serrano, A., \& Gallego-Izquierdo, T. (2019). Relación entre el dolor lumbar y el acortamiento isquitibial. Estudio de casos y $\begin{array}{lllll}\text { controles. European Journal of Podiatry, } & \text { 5(1), }\end{array}$ https://doi.org/10.17979/ejpod.2019.5.1.4360

Moreno-Quinchanegua, J. E. (2017). La fatiga, tipos, causas y efectos. Revista Digital: Actividad $\begin{array}{llllr}\text { Física } & y & \text { Deporte, } & 3(2), & \text { 87-95. }\end{array}$ https://revistas.udca.edu.co/index.php/rdafd/article/view/376

Mugueta-Aguinaga, I., Abasolo-Abrisqueta, E., Alonso-Alegre-Elorduy, I., Anakabe-Murelaga, A., Arbide- Ostolaiz, I., Bola-Saiz, P., Casal-Abad, V., Castañeda-Sánchez, I., Duque-Pascual, A., Flores-Ruiz, M. D., Goenaga-Echave, L., Gomez-Cuesta, A., López-Carballo, A., Peña-Vio, M., Pinedo-Crespo, A., Rodilla-Ojeda, I., Ruiz-Fuentes, A., \& Zabala-Acaiturri, A. (2015). Fisioterapeuta de empresa: Bienestar y progreso en el ámbito laboral. https://addi.ehu.es/bitstream/handle/10810/18834/DOSSIER\%20FPRL.pdf?sequence=1\&i sAllowed $=\mathrm{y}$

Pinchao, S., \& Aguado-Balanta, F. (2019). Actividad física en el entorno laboral como método de prevención en desordenes músculo esquelético [tesis de especialización, Universidad EAN]. Repositorio EAN. http://hdl.handle.net/10882/9557

Poder Ejecutivo Nacional. (2020, 20 de marzo). Decreto 297. Aislamiento social preventivo y obligatorio. Boletín Oficial de la República Argentina. https://www.boletinoficial.gob.ar/detalleAviso/primera/227042/20200320

Taboadela, C. H. (2007). Goniometría. Una herramienta para la evaluación de las incapacidades laborales. Asociart ART. https://aaot.org.ar/wpcontent/uploads/2019/12/Taboadela-Claudio-H-Goniometria-Eval-Incap-Laborales2007.pdf 
ElD Ergonomía, Investigación y Desarrollo, 3(3), 2021, 101-115

\section{Anexo}

Figura A1. Ficha de rehabilitación de hombros.

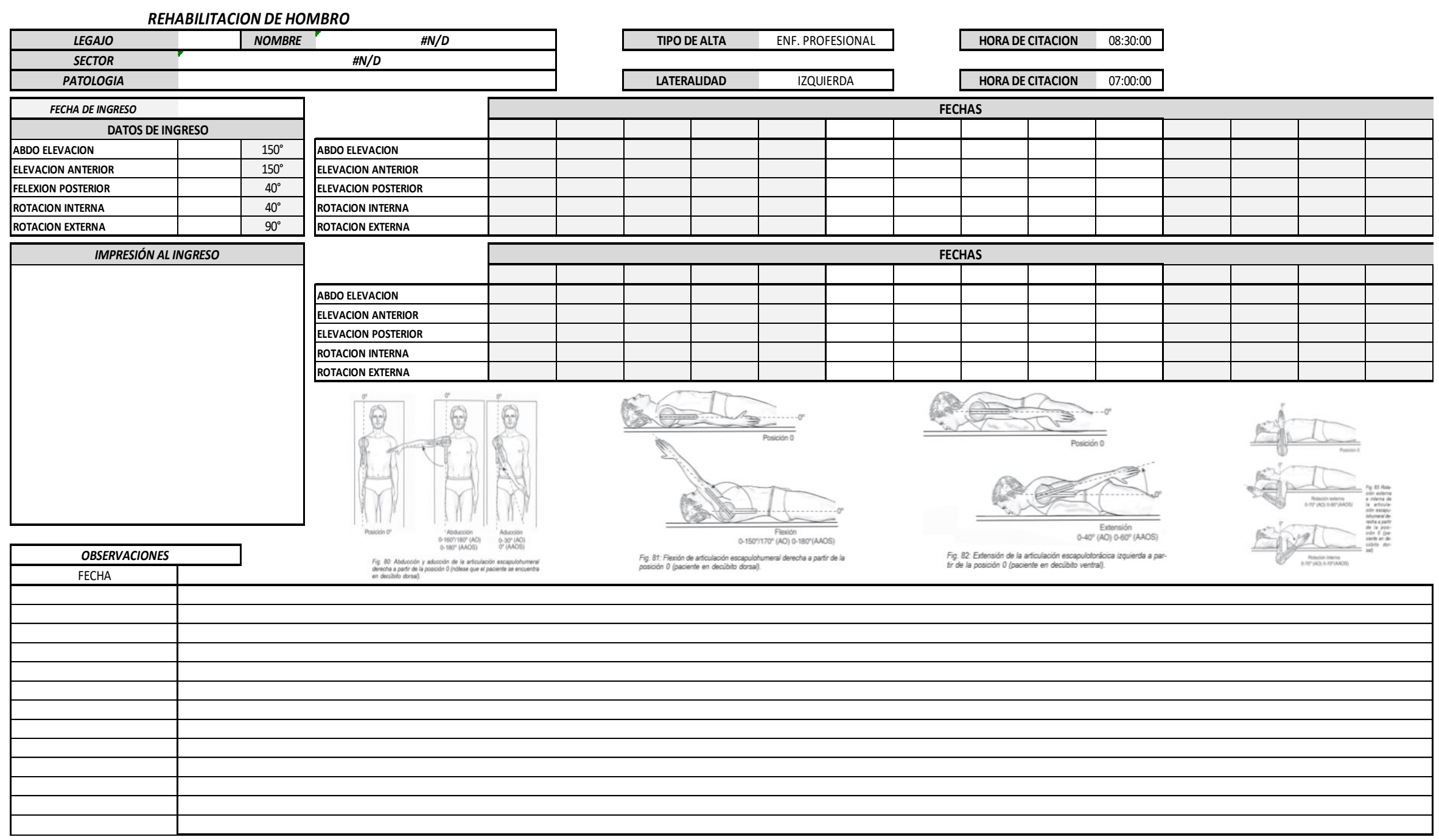

